\title{
R\&D for the local support and cooling channel for the ATLAS Pixel Insertable B-Layer (IBL)
}

\author{
Simone Coelli \\ Istituto Nazionale di Fisica Nucleare, Via Celoria 16, Milano I-20133, Italy. \\ On behalf of the ATLAS IBL Collaboration.
}

The Pixel Detector is the innermost tracking detector of the ATLAS experiment at the Large Hadron Collider (LHC) at CERN. A new internal pixel layer will be installed inside the existing three, in the space between the actual B-layer and a new smaller beam-pipe. The tracking performance will be enhanced due to a smaller radius, and the presence of a fourth layer will give higher redundancy and reliability to the Pixel Detector. After a description of this project and its relevant requirements, the current work on the design of local support and cooling channel is presented as well as the results of FEM analyses, of experimental prototyping, measurements and tests.

\section{Introduction: the ATLAS tracker}

ATLAS [1] is a general purpose particle physics experiment at the Large Hadron Collider (LHC). The trajectories of charged particles produced in the proton proton collisions are reconstructed by the Inner Detector, immersed in a 2 Tesla solenoidal magnetic field and consisting in three subdetectors, built with three different technologies: Pixel Detector, a silicon microstrip SemiConductor Tracker (SCT) and Transition Radiation Tracker (TRT). The Pixel Detector is the innermost part of the tracking system, placed immediately outside of the beam pipe and centered around the interaction point. It consists of three cylindrical layers at a distance of $5,9,12 \mathrm{~cm}$ from the beam line and of three discs per side, covering pseudorapidity $-2.5 \leq \eta \leq 2.5$. The Pixel Detector sensing devices are highly segmented so that charged particle trajectories can be accurately determined. It consists of 1744 modules, each of them with 47232 pixels and a total $80 \cdot 10^{6}$ channels. The pixel dimensions are $50 \times 400 \mu \mathrm{m}^{2}$. The detector cooling system has a $17 \mathrm{~kW}$ cooling power and uses evaporative fluorocarbon $\left(\mathrm{C}_{3} \mathrm{~F}_{8}\right)$ boiling at about $-27{ }^{\circ} \mathrm{C}\left(1.67 \operatorname{bar}_{\mathrm{A}}\right)$ in an integrated aluminum piping system.

\section{Requirements for an Insertable B-Layer for sLHC phase I}

The existing Pixel Detector B-Layer, the one at $5 \mathrm{~cm}$ from the beam line was foreseen to be replaced after few years of operation in order to cope to possible radiation damage. After $300 \mathrm{fb}^{-1}$ integrated luminosity, sensor and electronics degradation in the existing B-Layer are expected to sensibly reduce the detector efficiency. This is due to depletion voltage rise and charge trapping, giving lower collected charge and leakage current increase and then more noise and power dissipation. This could trigger a positive feedback effect, inducing a thermal runaway. Possible higher module operational temperature is an important issue. Potential leaks in the cooling loops could force to shutoff part of the detector. The Pixel Detector cannot be repaired in situ in case of failures of the cooling system, of optolinks or modules, and the subsequent inefficiencies would have a high impact on the physics potential of the experiment. The Pixel Detector package, with all the relevant structures, was designed to be disassembled using customized tools in a reversed sequence in order to repair the detector. However it has been understood that many reasons make this operation very difficult. The disinstallation, i.e. the extraction of the Pixel Detector package from its actual position in the centre 
of ATLAS, moving it to the surface clean room, is a delicate but necessary operation if one would access the innermost layer. It has to be noted that the actual $7.3 \mathrm{~m}$ long beryllium beam pipe is an inseparable part of the detector. It can be pulled out only after cutting one of its flanges, that are larger than the B-Layer, mounted around its central section. In addition, there is the complication to work in the ATLAS experimental site in an environment activated by the LHC beam collisions, and later on activated materials, during detector dismantling and reassembling. For all these reasons, a new option has been proposed: to construct a very low mass additional pixel layer, with about half the mass of the existing B-Layer. The current option is not a replacement, but rather an insertion of this new layer inside the current Pixel Detector, around a smaller beam pipe. In this way the installation time will be reduced. The presence of a fourth layer will give a bigger redundancy to the Pixel Detector, improve ATLAS tracking performance due to smaller radius of the tracker and the ATLAS physics performance will be enhanced.

\section{IBL local support design}

\subsection{Beam pipe extraction and insertion of the new integrated beam pipe and IBL}

The solution to insert a new IBL [2] rather than to replace the existing B-Layer implies that the space available in the detector to add something new is the volume of the actual beampipe, fully surrounded by the Pixel Package with a nominal clearance of $9 \mathrm{~mm}$. One option is therefore to substitute the present beampipe with a new smaller one and to use the free space between it and the existing envelope. The $29 \mathrm{~mm}$ radius of the current design beam-pipe should be reduced to $25 \mathrm{~mm}$. The present $7.3 \mathrm{~m}$ long section of the beam pipe will be cut, because its flange is too big to pass inside the existing Pixel Detector and will be extracted in situ. The new beampipe, with the IBL integrated on it, will then be inserted at its place. To simplify the insertion procedure and to carry the new detector load, a support carbon tube will be inserted and fixed before the installation of the new integrated beampipe and IBL.

\subsection{IBL support requirements}

The requirements for the IBL local support structure are here summarized. The most important point is the thermal management. As said before, under irradiation the dissipated power and therefore the temperature of the electronic components could increase, due to the rise of the leakage current with the temperature and the radiation damage (thermal runaway). This important effect is taken into account in the design by setting the cooling system evaporation temperature with a suitable margin, in order to ensure a good thermal performance of the stave. This leads to a design with an integrated cooling system, based on sub-zero temperature evaporating fluid. A fixed flow system feeds the cooling pipe through a capillary, then the fluid evaporates underneath the modules, extracting the thermal power. The actual system is based on boiling $\mathrm{C}_{3} \mathrm{~F}_{8}$, but the IBL will use boiling $\mathrm{CO}_{2}$, that allows a lower evaporation temperature, about $-40{ }^{\circ} \mathrm{C}$. Owing to the tight requirements in terms of material budget required by the ATLAS physics program, all this must be done with the lower possible mass. A composite structure made of carbon fibres provides the requested long term stability in terms of distortions induced by the loads, since it has an excellent stifness/weight ratio and an extremely low thermal expansion coefficient (CTE). The main loads are: weight, internal pressure and thermal loads induced by the cooling. It's desirable to have a low CTE for each part. The design of the stacking sequence of the carbon fibre pipe reduces this figure to about $1 \mathrm{ppm} /{ }^{\circ} \mathrm{C}$ on the longitudinal axis, a value nearly as low as that of the omega-shaped structural component made in a quasi-isotropic $(60 / 0 /-60)_{\mathrm{s}}$ composite, that is $0.2 \mathrm{ppm} /{ }^{\circ} \mathrm{C}$. The more stressed structural element is the cooling pipe. The design pressure is $10 \mathrm{MPa}$ using $\mathrm{CO}_{2}$. The pipe is designed to resist to a pressure test at $15 \mathrm{MPa}$ preserving the pipe tightness. Requirement of low mass means a low radiation length $\mathrm{X} / \mathrm{X}_{0}$. The target for the mechanical support is $0.5 \%$. The maximum allowed deformation is $0.1 \mathrm{~mm}$. There is the need of electro-chemical compatibility between the materials in contact. Radiation resistance and sta- 
bility under high doses have to be proven.

\subsection{Some IBL key numbers}

IBL will be made of 14 stave supports, $\sim 800 \mathrm{~mm}$ long, at a mean sensor radius of $33.25 \mathrm{~mm}$. Power of the cooling system should be $\sim 1.5 \mathrm{~kW}$. IBL has to survive to an integrated luminosity of $550 \mathrm{fb}^{-1}$.

\section{Innovation for the IBL}

\subsection{Cooling pipe technology}

Cooling pipe material could be chosen between the following materials: titanium, carbon fibre composite, stainless steel, aluminum. This last one has been discarded due to potential corrosion problems. Composite pipe needs to be qualified, but it is definitely compatible with the surrounding carbon based materials. R\&D is in progress to qualify both titanium pipe production, bending and welding procedures and the composite pipe technology. The thickness or the radiation length obtainable with these materials allow the lowest $\mathrm{X} / \mathrm{X}_{0}$ for the support. The most promising technology for the composite pipe production uses carbon fibre braiding at $+/-45^{\circ}$, infusion in the matrix resin, followed by degassing, drawing in a metallic mold and then curing at ambient temperature, using an inserted Teflon pull-out core. Work is in progress to study the behavior of the composite materials under repeated cooling to $-50{ }^{\circ} \mathrm{C}$ and irradiation with gamma rays and neutrons. Micro-cracking versus material strain dependence is under study, as well as the validation of the safety factor against internal pressure. With a composite pipe also the relevant joints need to be qualified. Some prototypes have been produced and tested of metallic-composite pipes glued together and over-braided with a reinforcing additional composite layer.

\subsection{Use of high conductive-low density carbon foams}

The conductive thermal contact between the cooling pipe and the heat power sources, frontend and sensor, is made through a light and high conductive carbon foam, to maintain the sensor temperature sufficiently low without adding too much material.

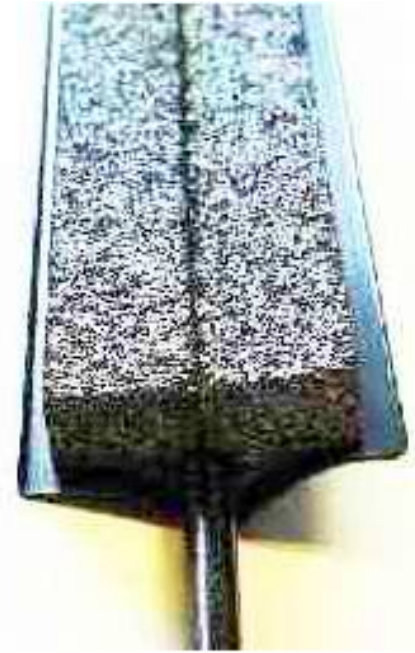

Figure 1. IBL stave prototype. The cooling pipe (outside diameter $3 \mathrm{~mm}$ ) is embedded into the carbon foam, supported by the omega-shaped structural carbon fibre composite.

The carbon foam hypothesis in this R\&D are: Poco Foam with density $0.55 \mathrm{~g} / \mathrm{cm}^{3}$ and conductivity $135 / 45 / 45 \mathrm{~W} / \mathrm{mK}$; otherwise Kopers KFOAM with density $0.245 \mathrm{~g} / \mathrm{cm}^{3}$ and conductivity $30 \mathrm{~W} / \mathrm{mK}$. The assembling procedure to make a stave with these materials has been proven. Stave prototypes built with titanium pipe or 1 or 2 carbon fibre pipes are under qualification.

\section{Finite Element Analysis: mathematical support to the stave design}

Numerical simulations are extensively performed during the design process to drive the engineering choices, together with prototype testing. Many different physical aspects are studied: thermal, structural, dynamic, and combination of them. For the composite materials special attention to the method is due, using the layered elements. The conductive thermal figure of merit of a stave configuration is used to evalu- 


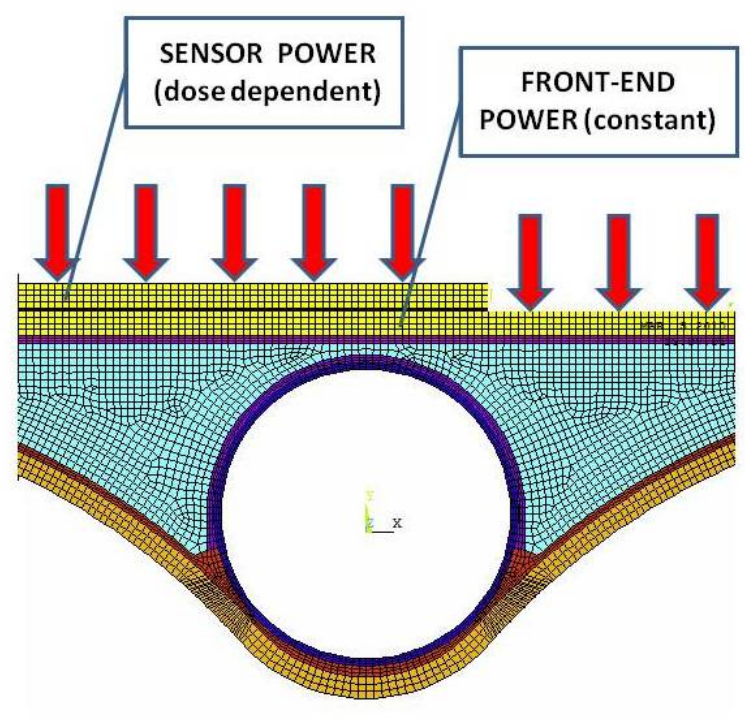

Figure 2. FEA example: a stave cross-section meshed for the thermal analysis.

ate its expected performances. This is defined as the maximum temperature difference between the inner surface of the cooling pipe and the sensor, divided by the heat flux on the surface of the foam. FEA has been used for the pressurized carbon fibre pipe design optimization with calculation of the Tsai-Hill safety factor and of the transverse strain value. This last is the parameter that controls the micro-crack growth in the ply, that has to be limited for the tightness assessment of the pipe. FEA has been used also for evaluation of CTE of various composites and for thermo-mechanical analysis, looking for induced deformation, stress and local effects. Dynamic analyses of the stave have been made checking the boundary conditions and giving the minimum frequency vibration-mode, to avoid possible resonances.

\section{Experimental measurements and tests}

At present a big effort is devoted to the qualification of the IBL stave via prototype production. A remarkable element is the cooling pipe, whose samples are checked with helium leak-rate measurement, before and after test pressurization. Burst pressure is measured to check the ultimate resistance of the pipe. Thermal performance measurements on real materials are made. Some stave prototypes are assembled in real cooling systems. To separate the thermal convective effects an environmental vacuum box is used. Thermal cycling is made 100 times from $+30{ }^{\circ} \mathrm{C}$ to $-50{ }^{\circ} \mathrm{C}$. Neutron irradiations in nuclear reactor are made to obtain the equivalent displacement per atom dose calculated by a Monte-Carlo method in a simulation of the radiation damage. This is done to estimate and check whether there are sensible effects in the matrix of the laminates. A dummy IBL has been prepared to simulate the sensor overheating during a simulated beam pipe bake-out. Cases of failure of some cooling loops are studied. FEA validation, particularly for the layered materials, is carried out through pull test on samples and making cooling tests on stave prototypes, for the thermal aspects. The transverse thermal conductivity $\left(\mathrm{K}_{\mathrm{t}}\right)$ of the composites and of the adhesives to be used is calculated from the following measured figures: density, specific heat and thermal diffusivity. This last one is measured through an innovative pulsed laserflash technique. As an example, for a carbon fibre pipe having outside diameter $3 \mathrm{~mm}$, thickness $0.3 \mathrm{~mm}$, made of T-300 fibres and 5052 Araldite matrix with an estimated $50 \%$ volume fiber and braided at $+/-45^{\circ}, \mathrm{K}_{\mathrm{t}}$ is $\sim 0.5 \mathrm{~W} / \mathrm{mK}$.

\section{REFERENCES}

1. The ATLAS Collaboration, The ATLAS experiment at the CERN Large Hadron Collider, JINST 3 S08003 (2008).

2. ATLAS Insertable B-Layer Technical Design Report and references therein, CERN-LHCC2010-013. 